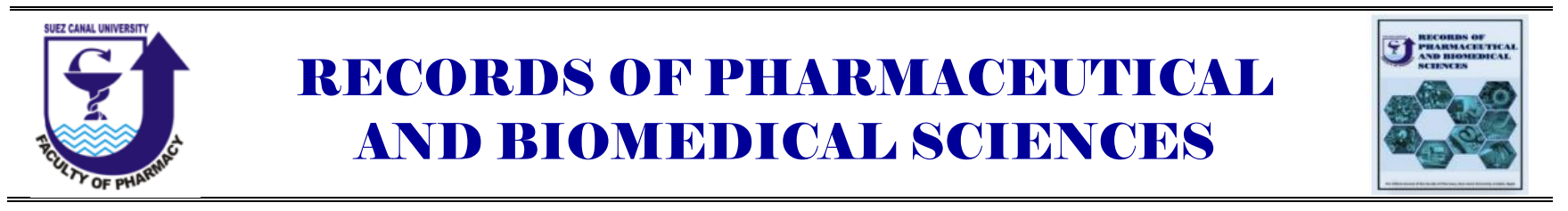

\title{
Drug Delivery from Microsponges: A Review Article
}

\author{
Radwa M. A. Abd-Elala ${ }^{a}$, Ghada H. Elosaily ${ }^{a, b}$, El-Sayed Khafagy ${ }^{c, d}$, Yasser Mostafa ${ }^{e}$, Shadeed Gad * \\ ${ }^{a}$ Department of Pharmaceutics, Modern University for Technology \& Information (MTI), Cairo, Egypt,. \\ ${ }^{b}$ Department of Pharmaceutics and Industrial Pharmacy, Faculty of Pharmacy, Al-Azhar University, Cairo, \\ Egypt, ${ }^{c}$ Department of Pharmaceutics and Industrial Pharmacy, Faculty of Pharmacy, Suez Canal \\ University, Ismailia, Egypt 41522, ${ }^{d}$ Department of Pharmaceutics, College of Pharmacy, Prince Sattam Bin \\ Abdulaziz University (PSAU), Al-Kharj 1142, Kingdom of Saudi Arabia (KSA), ${ }^{e}$ Department of \\ Pharmacology and Toxicology, Faculty of Pharmacy, Suez Canal University, Ismailia, Egypt
}

Received on: 04. 02. 2020

Revised on: 25. 03. 2020

Accepted on: 01. 04. 2020

Correspondence Author:

Tel: +201003934422

E-mail address:

shadedabdelrahman@pharm.suez.edu.eg

\begin{abstract}
Microsponges are micro-porous particles, used mainly for topical and recently for oral administration. Microsponges have many advantages which make it a versatile drug delivery system. Microsponges can suspended or entrap a wide variety of substances and then be incorporated into a formulated product such as a gel, cream, liquid, or powder. Moreover, they may enhance stability, reduce side effects and modify drug release related to its porous structure which allows the active ingredient to sustain over time. The aim of this article is to provide details about microsponges including the method of preparation, characterization, mechanism of drug release from microsponges, different formulation and process factors, and a few applications about microsponges which are either proven or under research.
\end{abstract}

Keywords: Microsponges, drug delivery, Quasi-emulsion solvent diffusion, porous particles, topical applications

\section{Introduction}

With advances in biotechnology, genomics, and combinatorial chemistry, a wide variety of new, more potent and specific therapeutics are being created. Because of common problems such as low solubility, high potency, and/or poor stability of many of these new drugs, the means of drug delivery can impact efficacy and potential for commercialization as much as the nature of the drug delivery. Indeed, different drug delivery systems designed to provide a therapeutic agent in the needed amount, at the right time, to the proper location in the body, in a manner that optimizes efficacy, increase compliance and minimize side effects.

Microsponges are polymeric delivery system composed of porous microspheres which having a particle size range of 5-300 $\mu \mathrm{m}$ with a capability to entrap a wide range of active ingredients and are used as a carrier for topical drug delivery, as shown in Figure 1 (Junqueira and Bruschi, 2018). Microsponges are tiny sponge-like spherical particles that consist of a myriad of interconnecting 
avoids within a non-collapsible structure having a large porous surface (Junqueira and Bruschi, 2018, Orlu et al., 2006). The microsponge technology was developed by Won in 1987 and the original patent was assigned to Advanced Polymer Systems, Inc. This company developed a large number of variations of the technique and applied those to cosmetic as well as over the counter and prescription pharmaceutical products. At the present time, this interesting technology has been licensed to Cardinal Health, Inc. for use in topical products (Shah and Shah, 2014). Microsponges are designed to deliver a pharmaceutically active ingredient efficiently at the minimum dose and also to enhance stability, reduce side effects, and modify drug release profiles. These attributes have been successfully demonstrated in the FDA-approved Retin-A Micro ${ }^{\circledR}(0.1 \%$ or $0.04 \%$ tretinoin $)$ and Carac $(0.5 \%$ 5-fluorouracil) products for acne treatment and actinic keratoses, respectively (Shah and Shah, 2014). Microsponges are considered a good candidate to deliver different active ingredients to different drug delivery systems related to its advantages such as; microsponges are biologically safe (non-allergic, non-toxic, nonirritant and non-mutagenic) (D'souza and More, 2008), have the capacity to absorb or load a high degree of active materials into the particle or into its surface (Embil and Nacht, 1996), stable over a pH range of $1-11$ and temperature up to $130{ }^{\circ} \mathrm{C}$, selfsterilizing as average pore size is $0.25 \mu \mathrm{m}$ where bacteria cannot penetrate, able to entrap various ingredients in a single microsphere with size 10-25 $\mu \mathrm{m}$, microsponges are chemical, physical, and thermally stable, have a good compatibility with various vehicles and ingredients, provide a continuous action up to $12 \mathrm{~h}$, which means that these particles can extend drug release, so improve product elegancy, and payload is up to $50-60 \%$, with an advantage of cost effectiveness over the other formulations (Joshi and RajandeepKaur, 2016).

\section{Preparation of microsponges}

Drug loading in microsponges can take place in two ways, one-step process (Liquid-liquid suspension polymerization) or by two-step process (Quasiemulsion solvent diffusion method) which are based on physicochemical properties of a drug. If the drug is typically an inert non-polar material, it will create a porous structure and this called Porogen. Porogen drug, which neither hinders the polymerization nor becomes activated by it and stable to free radicals are entrapped by the one-step process (Singhvi et al., 2019, Kaity et al., 2010).

\subsection{Liquid-liquid suspension polymerization}

In general, the monomers are first dissolved along with active ingredients in a suitable solvent solution of monomer. Then, it suspended with agitation in an aqueous phase, usually containing additives, such as surfactants and dispersants, to promote suspension. Once the suspension is established with discrete droplets of the desired size, polymerization is initiated by activating the monomers either by catalysis or by increasing temperature or irradiation. As the polymerization process continues, a spherical structure is produced containing thousands of microsponges bunched together like grapes, forming interconnecting reservoirs (Hainey et al., 1991). Once the polymerization completes the solid particles are washed and stored, Figure 2.

\subsection{Quasi-emulsion solvent diffusion}

When the drug is sensitive to the polymerization conditions, the two-step process is used. In general, an active substance with a different drug: polymer ratios were dissolved in the organic solvent (inner phase). Then, the obtained solution was poured into a polyvinyl alcohol solution (external phase) with continuous stirring in order to evaporate the solvent, then the mixture is filtered to separate the microsponges, Figure (3). The obtained microsponges are dried and stored in desiccators to ensure the complete removal of residual content (Orlu et al., 2006).

\section{Mechanism of drug release from microsponges}

Drug release from microsponges occurred over time in response to one or more external triggers such as (temperature, pressure, $\mathrm{pH}$, and solubility) (Singhvi et al., 2019).

\subsection{Pressure}

Rubbing or pressure applied can release active ingredients from microsponge onto the skin.

\subsection{Temperature}

The flow rate of the active ingredient from microsponges was affected by temperature change. With increased skin temperature, the flow rate is also increased and therefore release is also enhanced. 


\section{3.pH}

Triggering the $\mathrm{pH}$-based release of the active ingredient can be occurred by modifying the coating on the microsponges.

\subsection{Solubility}

Microsponges loaded with hydrophilic active ingredients such as antiseptics and antiperspirants will be released in the presence of aqueous media. The release can also be achieved by diffusion but taking into consideration, the partition coefficient of the ingredient between the microsponges and the external system.

\section{Physical characterization of microsponges}

\subsection{Production yield (PY)}

The PY (\%) of the TMM-loaded microsponges was determined by calculating precisely the beginning weight of the compounded materials and the ultimate weight of the microsponges, according to the following equation (Moin et al., 2016).

PY (\%)

$=\frac{\text { The ultimate weight of microsponges }(\mathrm{mg})}{\text { (The beginning weight }(\text { polymer }+ \text { drug })(\mathrm{mg}))}$

\subsection{Particle size}

Particle size examination of microsponges could be carried out by laser light diffractometer or any suitable methods. The values (d50) can be expressed for all formulations as a mean size range. Particles larger than $30 \mu \mathrm{m}$ can impart gritty feeling and hence particles of size 10 to $25 \mu \mathrm{m}$ are preferred to use in a final topical formulation.

\subsection{Entrapment Efficiency (E.E.)}

The obtained loaded-microsponges were mixed with a suitable amount of phosphate buffer saline (PBS, $\mathrm{pH}=7.4$ ) or any other suitable solvent to allow drug extraction with continuous shaking. Then, the drug content was determined by measuring the absorbance at the predetermined wavelength of the drug. The E.E. (\%) was calculated related to the following equation:

$$
\begin{aligned}
& \text { E. E. (\%) } \\
& =\frac{\text { The actual drug content in microsponges }}{\text { Theoretical drug content }} \times 100
\end{aligned}
$$

\subsection{Morphology and surface topography of microsponges}

Microsponges can be coated with gold-palladium under an argon atmosphere at room temperature. Then the scanning electron microscopy was used to study the surface morphology of the microsponges (Orlu et al., 2006).

\subsection{Compatibility studies}

Thin-layer chromatography and Fourier Infra-red spectroscopy can be used to estimate the compatibility between drugs and excipients. Drug crystallinity can be studied by X-ray diffraction and Differential Scanning Colorimetry (Moin et al., 2016).

\subsection{Dissolution studies}

Dissolution apparatus USP with certain modifications was used for studying the dissolution profile of the loaded microsponges. The dissolution medium is selected by considering the solubility of the drug to ensure sink conditions. After different time intervals, samples were withdrawn from the dissolution medium and analyzed by a suitable analytical method. Then, the kinetics studies were done by fitting the in-vitro drug release data to different models to determine the kinetics of drug release from the prepared microsponges (Bruschi, 2015), according to the following equations:

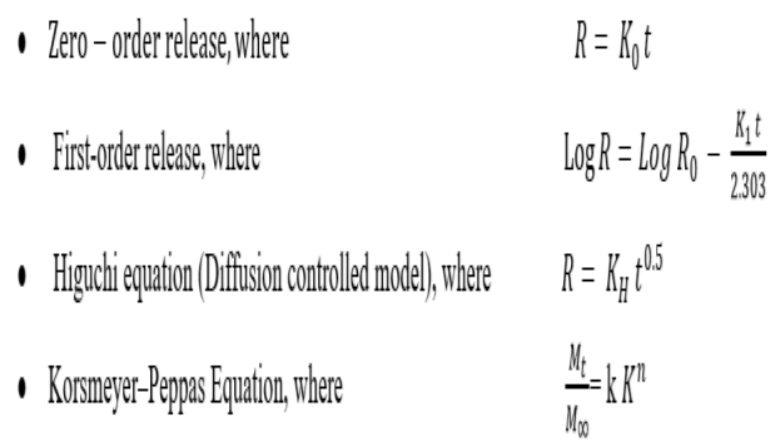

\section{Effect of different formulation and process variables on physicochemical properties of microsponges (Aloorkar et al., 2012, Pandey et al., 2013)}

\subsection{Polymer or monomer composition}

Microsponge size, drug loading and polymer design regulate drug release from microsponges. The polymer composition can affect the partition coefficient of the entrapped drug between the vehicle and the microsponge system. So, it has a 
direct impact on the release rate of the entrapped drug. Polymer selection is dictated both by characteristics of the drug ultimately to be entrapped and by the vehicle into which it will be dispersed. Polymers with varying electrical charges or degrees of hydrophobicity may be prepared to confer flexibility in the release of the drug. Several polymer combinations will be screened for their suitability with the drugs by studying their drug release profile.

\subsection{Effect of the drug to the polymer ratio}

When the amount of polymer is kept constant while the ratio of drug to polymer is modified, the drug loading capacity is not much affected by this change, however, the production yield can extremely be altered from minimum ratio to maximum one. In addition, the particle size is also affected by the change of the drug to the polymer ratio. It has been examined that when the drug amount is increased, the particle size is also enhanced by the formed microsponges.

\subsection{Effect of stirring rate}

Stirring rate is one of the important process parameters to obtain microsponges particle with small particle size and high entrapment efficiency. During solvent evaporation, the droplets are solidified to produce particles and the drug is adsorbed on the surface and/or entrapped within the polymer matrix of these particles. Moreover, during the transition of droplets to form particles, the drug may have a tendency to diffuse out into the external phase. Thus, it is important to control the solidification rate of the droplets in order to achieve small particle size and high entrapment efficiency.

\section{Applications of microsponges as drug delivery system}

Microsponges are mostly applicable for topical and recently for oral administration as well as biopharmaceutical delivery. The following are a few uses, which are either proven or under research.

\subsection{Burn wound therapy}

Silver sulfadiazine-loaded microsponges were developed using water in oil in water Quasiemulsion solvent diffusion method. The prepared loaded microsponges were incorporated in the gel base, which enhanced the efficacy of the drug by reducing the cytotoxicity towards the keratinocytes and fibroblasts without altering the antimicrobial properties. Microsponges have an ability to improve the delivery of silver sulfadiazine to burn wounds and minimizing cytotoxicity towards host cells (Kumar and Ghosh, 2017).

\subsection{Anti-fungal}

Eberconazole nitrate-loaded microsponges were developed using the Quasi-emulsion solvent diffusion method. The obtained microsponges were dispersed in a gel, and an in-vivo skin deposition study was demonstrated and showed that the loaded microsponges had fourfold higher retention in the stratum corneum layer compared with the commercial cream (Bothiraja et al., 2014).

\subsection{Anti-acne}

Retinoic acid-loaded microsponges were developed and tested for drug release and anti-acne efficacy. Statistically significant, greater reductions in inflammatory and non-inflammatory lesions were obtained with tretinoin entrapped in the microsponge.

\subsection{Colon-specific drug targeting for treating rheumatoid arthritis}

The controlled delivery of flurbiprofen was conducted by using a commercial Microsponge ${ }^{\circledR}$ 5640 system. In-vitro studies exhibited that compression-coated colon-specific tablet formulations started to release the drug for $8 \mathrm{~h}$, corresponding to the proximal colon arrival time. This is due to the addition of the enzyme, following a modified release pattern, while the drug release from the colon-specific formulations prepared by pore plugging the microsponges showed an increase for $8 \mathrm{~h}$, which was the point of time when the enzyme addition was made (Orlu et al., 2006).

\subsection{Anti-glaucoma}

Stable acetazolamide microsponges were successfully prepared by the Quasi-emulsion solvent diffusion technique. Ex-vivo studies exhibited that acetazolamide microsponges in-situ gel formulation could be successfully used for topical ocular administration for the treatment of glaucoma, and avoiding systemic side effects compared to the oral acetazolamide orally (Obiedallah et al., 2018).

\subsection{Anti-cancer}

Microsponges based topical gel formulation of 5Fluorouracil (5-FU) for the treatment of skin cancer with enhanced skin deposition and reduced skin 
irritation potential. Brunauer-Emmett-Teller analysis demonstrated higher surface area and pore volume of developed microsponges' formulation. The optimized formulation showed better thixotropic and texture properties compared to the commercial cream formulation, used as the control for comparison purposes.

Further, the optimized formulation demonstrated a 5.5-fold increase in skin deposition documented via in-vivo local bioavailability study, with a significant reduction in skin irritation compared to the commercial formulation.

Hence, the developed microsponges based formulation seems to be a viable alternative with enhanced topical delivery of 5-FU as compared to the commercial formulation (Jain et al., 2019).

\section{Conclusion}

The microsponge delivery system is a unique technology for the controlled release of macroporous particles, loaded with active ingredients. Microsponges offer a potential decrease in side effects while maintains its therapeutics effect. In addition, microsponges improve stability, increase elegance, and enhance formulation flexibility. Previous studies were done and confirmed that microsponges are non-irritant, non-allergenic, non-mutagenic, and non-toxic. This technology is being used currently in cosmetics, sunscreens, and prescription products. Therefore, the microsponges based drug delivery technology is likely to become a valuable drug delivery matrix substance for various therapeutic applications in the future

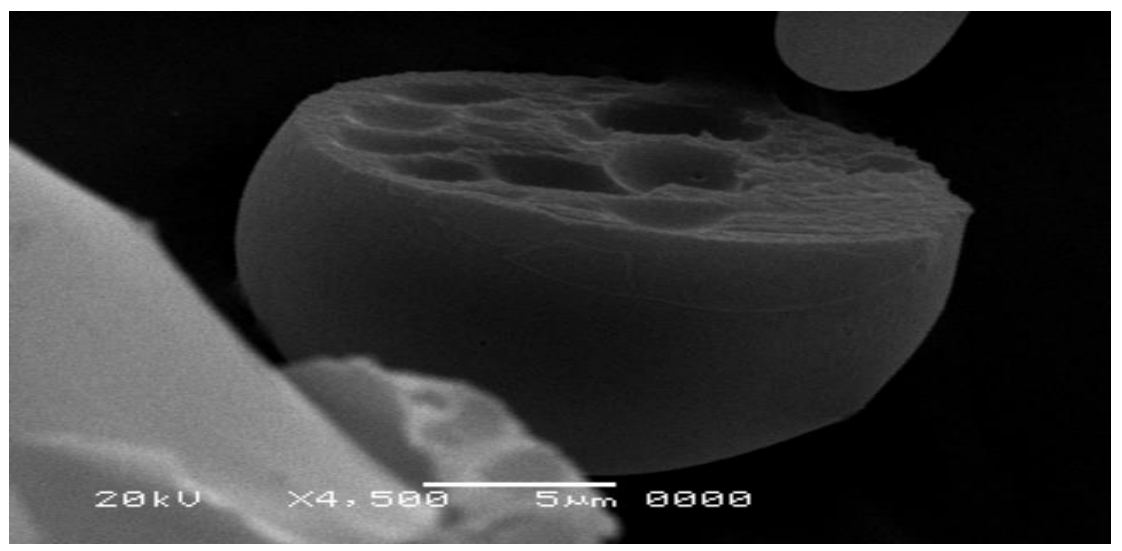

Fig. (1): Scanning electron micrograph of microsponges

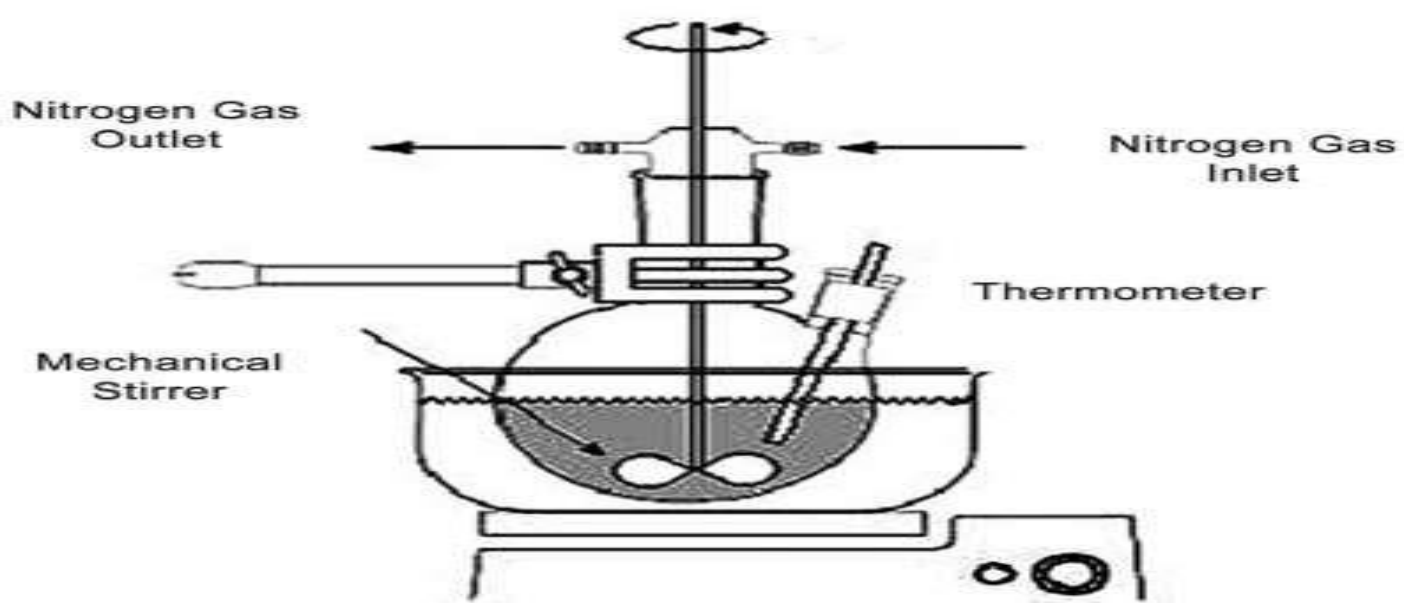

Fig. (2): Schematic representation of microsponges preparation by liquid-liquid suspension polymerization (Kaity et al., 2010). 


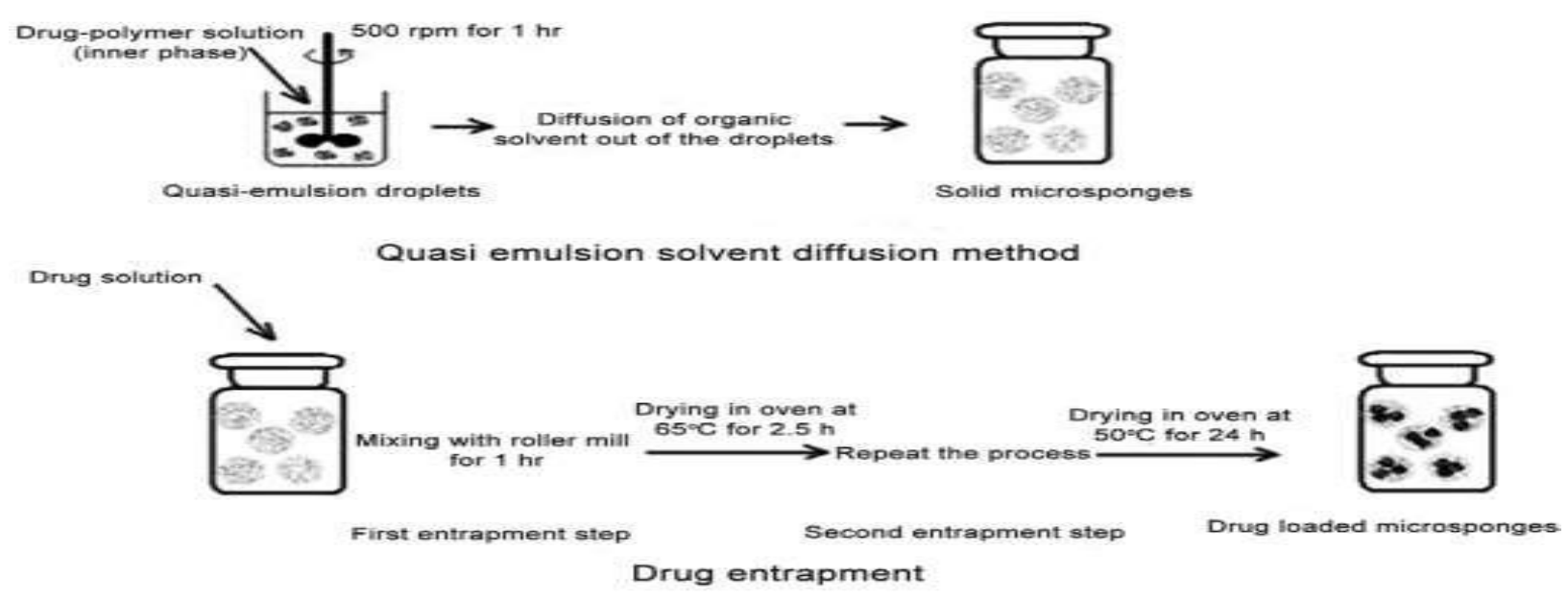

Fig. (3) : Schematic representation of microsponges prepared by the Quasi-emulsion solvent method (Kaity et al., 2010).

\section{Conflict of interest}

The authors report no declaration of conflict of interest.

\section{References}

ALOORKAR, N., KULKARNI, A., INGALE, D. \& PATIL, R. 2012. Microsponges as innovative drug delivery systems. Int J Pharm Sci Nanotechnol, 5, 1597-1606.

BOTHIRAJA, C., GHOLAP, A. D., SHAIKH, K. S. \& PAWAR, A. P. 2014. Investigation of ethyl cellulose microsponge gel for topical delivery of eberconazole nitrate for fungal therapy. Ther Deliv, $5,781-794$.

BRUSCHI, M. L. 2015. Strategies to modify the drug release from pharmaceutical systems, Woodhead Publishing.

D'SOUZA, J. I. \& MORE, H. N. 2008. Topical antiinflammatory gels of fluocinolone acetonide entrapped in eudragit based microsponge delivery system. Res J Pharm Technol, 1, 502-506.

EMBIL, K. \& NACHT, S. 1996. The microsponge ${ }^{\circledR}$ delivery system (MDS): a topical delivery system with reduced irritancy incorporating multiple triggering mechanisms for the release of actives. $J$ Microencapsul, 13, 575-588.

HAINEY, P., HUXHAM, I., ROWATT, B., SHERRINGTON, D. \& TETLEY, L. 1991. Synthesis and ultrastructural studies of styrene- divinylbenzene polyhipe polymers. Macromol, 24, 117-121.

JAIN, S. K., KAUR, M., KALYANI, P., MEHRA, A., KAUR, N. \& PANCHAL, N. 2019. Microsponges enriched gel for enhanced topical delivery of 5-fluorouracil. J Microencapsul, 1-34.

JOSHI, G. \& RAJANDEEPKAUR, H. K. 2016. Microsponges: a novel drug delivery system. IRJPBS, 3, 01-11.

JUNQUEIRA, M. V. \& BRUSCHI, M. L. 2018. A review about the drug delivery from microsponges. AAPS PharmSciTech, 19, 1501-1511.

KAITY, S., MAITI, S., GHOSH, A. K., PAL, D., GHOSH, A. \& BANERJEE, S. 2010. Microsponges: a novel strategy for drug delivery system. J Adv Pharm Technol Res, 1, 283.

KUMAR, P. M. \& GHOSH, A. 2017. Development and evaluation of silver sulfadiazine loaded microsponge based gel for partial thickness (second degree) burn wounds. Eur J Pharm Sci, 96, 243-254.

MOIN, A., DEB, T. K., OSMANI, R. A. M., BHOSALE, R. R. \& HANI, U. 2016. Fabrication, characterization, and evaluation of microsponge delivery system for facilitated fungal therapy. $J$ Basic Clin Pharm., 7, 39.

OBIEDALLAH, M. M., ABDEL-MAGEED, A. \& ELFAHAM, T. H. 2018. Ocular administration of acetazolamide microsponges in situ gel 
formulations. Saudi Pharm J, 26, 909-920.

ORLU, M., CEVHER, E. \& ARAMAN, A. 2006. Design and evaluation of colon specific drug delivery system containing flurbiprofen microsponges. Int J Pharm, 318, 103-117.

PANDEY, P., JAIN, V. \& MAHAJAN, S. 2013. A review: microsponge drug delivery system. Int $J$ Biopharm, 4, 225-230.
SHAH, C. N. \& SHAH, D. P. 2014. Microsponges: a revolutionary path breaking modified drug delivery of topical drugs. Int J Pharm Res, 6, 1-13.

SINGHVI, G., MANCHANDA, P., HANS, N., DUBEY, S. K. \& GUPTA, G. 2019. Microsponge: an emerging drug delivery strategy. Drug Develop Res, 80, 200-208. 